\title{
Emergence of new oral antithrombotics: a critical appraisal of their clinical potential
}

\author{
Michael Rud Lassen' \\ Volker Laux ${ }^{2}$ \\ 'Department of Orthopaedics \\ and Clinical Trial Unit, Nordsjællands \\ Hospital Hørsholm, University \\ of Copenhagen, Hørsholm, Denmark; \\ ${ }^{2}$ Thrombosis Research, Bayer Schering \\ Pharma, Wuppertal, Germany
}

\begin{abstract}
In Western countries, venous thromboembolism (VTE) is a widespread and serious disorder, with hospital admission rates that appear to be increasing. Current anticoagulant therapies available for the prevention and treatment of VTE have several drawbacks that make them either difficult to manage effectively, due to a need for careful monitoring to control coagulation, or, in the case of parenterally administered agents, inconvenient for long-term use. To address some of these issues, new anticoagulants are in clinical development that can be orally administered and directly target specific factors in the coagulation cascade. This article reviews the rationale behind development of these novel agents and provides a critical appraisal of their clinical potential. In addition, the impact that the introduction of such agents into clinical practice would have is discussed from the patient perspective.
\end{abstract}

Keywords: antithrombotic agents, venous thromboembolism, Factor Xa, thrombin

\section{The history of antithrombotics}

Anticoagulants are recommended for the prevention and treatment of venous thromboembolism (VTE), and the prevention of thromboembolic events in patients with chronic conditions such as atrial fibrillation (AF) (Buller et al 2004; Geerts et al 2008), or in patients with mechanical heart valves. For the prevention of VTE, the American College of Chest Physician (ACCP) guidelines recommend that extended thromboprophylaxis should be given to patients for up to 35 days (grade 1A) following total hip replacement (THR) and for at least 10 days after total knee replacement (TKR) (grade 1A) (Geerts et al 2008). Currently available anticoagulants comprise the heparins - unfractionated heparin (UFH) and the low molecular weight heparins (LMWHs), eg enoxaparin, tinzaparin, dalteparin - the vitamin K antagonists (VKAs), including warfarin, and the synthetic pentasaccharide fondaparinux. Although effective, these agents have significant limitations (Table 1).

UFH, developed more than 60 years ago (Hirsh et al 2007), requires parenteral administration, making it inconvenient for use outside the hospital setting. It also requires coagulation monitoring and is associated with heparin-induced thrombocytopenia (HIT) and osteopenia (Hirsh et al 2001). The LMWHs, developed in the 1980s, overcame some of the drawbacks associated with UFH: they do not require monitoring and have a substantially lower risk of HIT compared with UFH (Warkentin et al 1995). However, LMWHs are administered by subcutaneous injection, and accumulation can occur in patients with renal impairment (Hirsh et al 2004).

VKAs have been in use in humans for more than 50 years and are currently the only oral anticoagulants available. The utility of VKAs is limited by the difficulty of managing them, the requirement of frequent monitoring and the necessity for dose adjustment to limit the adverse consequences of a narrow therapeutic window, multiple food and drug interactions, and variable pharmacology. These qualities, in addition to 
the bleeding risk and other adverse effects, may contribute to the frequent underuse of warfarin, especially in elderly patients (Ansell et al 2004; Dantas et al 2004). In addition, VKAs have a slow onset of action. This can be a particular problem in VTE treatment, when the patient is at immediate risk of thrombus growth. In this situation, bridging therapy initiated with parenteral anticoagulants with a fast onset of action is necessary.

Fondaparinux, approved for use in the US in 2001 and Europe in 2002, has been shown to be relatively safe and effective in a variety of indications. However, like the heparins, it requires parenteral administration, which can be inconvenient when long-term use is necessary. Moreover, fondaparinux can also accumulate in patients with renal impairment due to renal elimination kinetics. Clearly, there is an unmet need for a convenient, safe antithrombotic agent that can be administered orally and does not require frequent monitoring or dose adjustment.

\section{Current focus of antithrombotic development}

The rationale behind the development of antithrombotics is based on an understanding of the coagulation cascade (Figure 1). The coagulation cascade can be initiated via either the intrinsic or extrinsic pathways. Initiation of the intrinsic coagulation cascade occurs when prekallikrein, high-molecular-weight kininogen, Factor XI, and Factor XII are exposed to a negatively charged surface, eg, phospholipids of circulating lipoprotein particles or bacterial surfaces. This is termed the contact phase and results in the conversion of prekallikrein to kallikrein, which in turn catalyzes the activation of Factor XII to activated Factor XII (FXIIa). FXIIa promotes the activation of Factor XI to FXIa, causing the release of bradykinin from high-molecular-weight kininogen. Factor IX is a proenzyme that contains vitamin $\mathrm{K}$-dependent $\gamma$-carboxyglutamate residues, whose serine protease activity is activated following $\mathrm{Ca}^{2+}$ binding to the $\gamma$-carboxyglutamate residues. In the presence of $\mathrm{Ca}^{2+}$, FXIa catalyzes the activation of Factor IX to FIXa. FIXa catalyzes the activation of Factor X to FXa, through interaction with the protein cofactor VIII (intrinsic tenase).

The extrinsic coagulation cascade is initiated following vascular injury by exposure of tissue factor (TF) to circulating plasma coagulation factors. TF and activated Factor VII (FVIIa; extrinsic tenase) catalyze the conversion of Factor $\mathrm{X}$ to FXa. The TF/FVIIa complex also catalyzes the activation of Factor IX of the intrinsic pathway, which in turn catalyzes the activation of Factor X. FXa, the point where the two coagulation cascades meet, catalyzes the activation of prothrombin (Factor II) to form thrombin (Factor IIa). The activation of thrombin occurs on the surface of activated platelets and requires formation of a prothrombinase complex. This complex is composed of the platelet phospholipids, phosphatidylinositol and phosphatidylserine, $\mathrm{Ca}^{2+}$, Factors $\mathrm{Va}$ and $\mathrm{Xa}$, and prothrombin. Thrombin catalyzes the conversion of fibrinogen (Factor I) to fibrin (Factor Ia) and fibrin forms a mesh that, in conjunction with the platelets, plugs the break in the vessel wall. Thrombin also catalyzes the activation of Factor XIII, consequently stabilizing the fibrin network by forming crosslinks (Davie 1995).

Conventional therapies (ie VKAs, UFH, and LMWHs) act on multiple targets within the coagulation cascade (Hawkins 2004). VKAs inhibit the vitamin-K-dependent $\gamma$-carboxylation of the clotting factors prothrombin and Factors VII, IX and X (McBride 2005). UFH and LMWHs potentiate the inhibitory action of antithrombin on thrombin

Table I Limitations of current anticoagulants

\begin{tabular}{lll}
\hline Anticoagulant & Limitations & Consequences \\
\hline UFH & Parenteral mode of administration & Inconvenient for long-term use \\
& Unpredictable anticoagulant effect due to unspecific binding & Regular monitoring of aPTT required \\
& Risk of HIT & Monitoring of platelet count required \\
LMWH & Parenteral mode of administration & Inconvenient and expensive for long-term use \\
& Risk of HIT & Monitoring of platelet count required \\
VKAs & Unpredictable anticoagulant effect & Regular monitoring and dose adjustment required \\
& Slow onset of action and narrow therapeutic window & to ensure patients stay within target INR (usually 2-3) \\
Fondaparinux & Food and drug interactions & Risk of adverse events (for example, bleeding) \\
\hline
\end{tabular}

Abbreviations: UFH, unfractionated heparin; LMWH, low-molecular-weight heparin;VKAs, vitamin K antagonists; aPTT,activated partial thromboplastin time; HIT, heparin-induced thrombocytopenia; INR, international normalized ratio. 


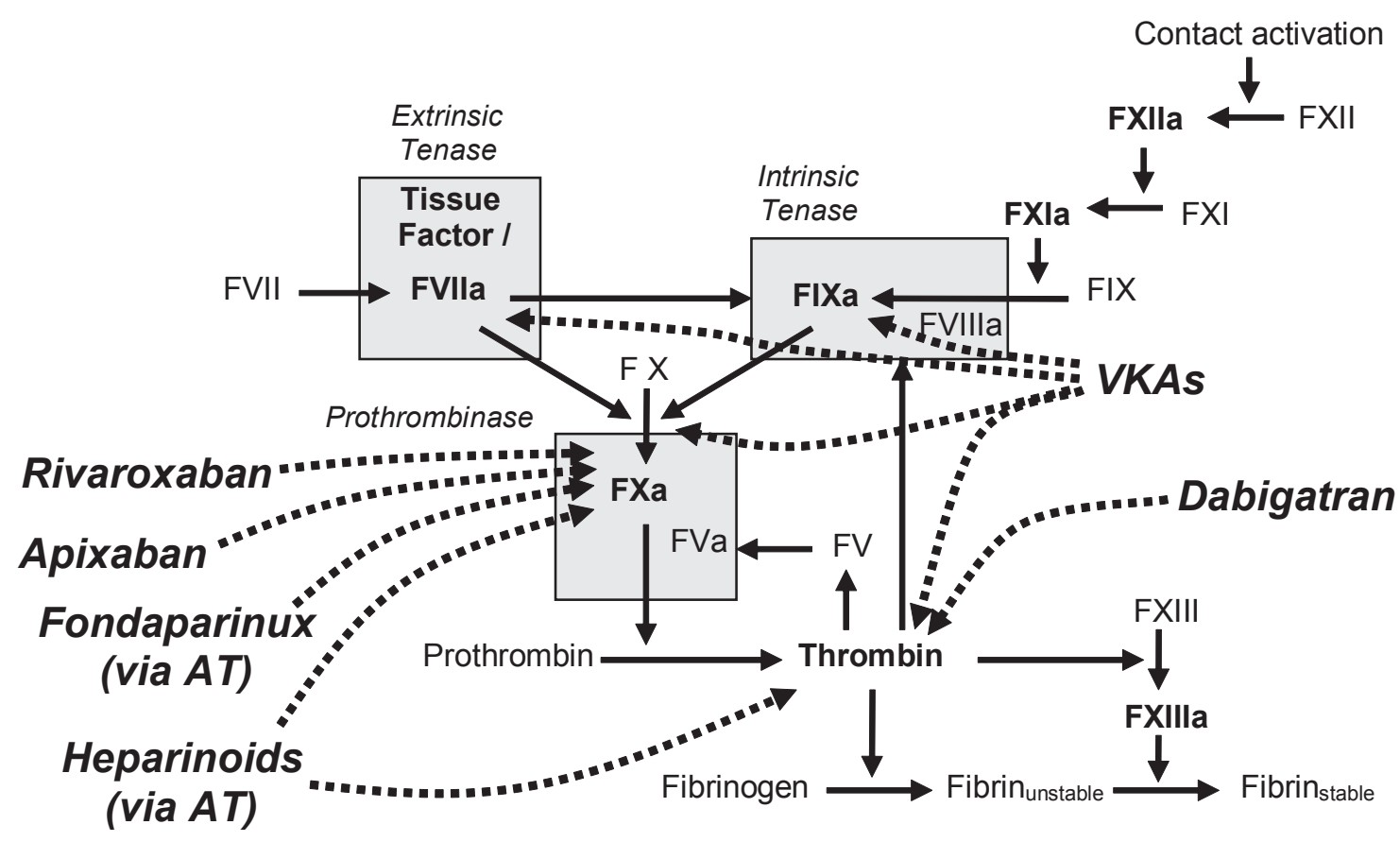

Figure I The coagulation cascade.

Abbreviations: VKAs, vitamin $\mathrm{K}$ antagonists; $\mathrm{AT}$, antithrombin.

and FXa, and also induce the release of TF pathway inhibitor from endothelial cells, further enhancing their anticoagulant activity (Hirsh et al 2007). The unpredictable anticoagulation patterns sometimes observed with VKAs and UFH could in part be explained by their action on multiple factors, because each factor targeted has a different half-life. Furthermore, thrombin formation is individualized due to genetic factors that are still not fully understood. In addition, conventional therapies are unable to antagonize the effects of thrombin bound to the clot, even though clot-bound thrombin retains enzymatic activity. Because thrombin potentiates its own generation via feedback stimulation of FV, FVIII, and FIX, this creates the potential for therapeutic failure (McBride 2005).

In an attempt to render the effects of anticoagulants more predictable than the VKAs and UFH, recent research efforts have focused primarily on the direct inhibition of a single coagulation factor, namely thrombin and $\mathrm{FXa}$ - two serine proteases with key functions in the coagulation cascade (Ansell 2007). Thrombin is a procoagulant but also plays an important role in anticoagulation and anti-inflammation via thrombin-thrombomodulin-mediated activation of protein $\mathrm{C}$ (Linder et al 1999). Thrombin also promotes inflammation and cellular proliferation (Esmon et al 1993; Tarzami et al 2006; Vesey et al 2005). The early direct thrombin inhibitors (DTIs) bivalirudin and argatroban, which provided proof of concept for direct thrombin inhibition, are still in use today. However, due to their specific pharmacokinetic and pharmacodynamic properties, they are used only in specific patient populations, eg in patients undergoing percutaneous coronary intervention or in patients with HIT. Ximelegatran (AstraZeneca) was the first oral DTI developed and was a prodrug of the active-site-directed thrombin inhibitor, melagatran (Gustafsson et al 2001). Ximelagatran was shown to be effective for the prevention and treatment of VTE in several phase II and phase III clinical trials: METHRO III (Eriksson et al 2003b), EXPRESS (Eriksson et al 2003a), EXULT A and B (Colwell, Jr. et al 2003; Francis et al 2003), and THRIVE II and III (Fiessinger et al 2005; Schulman et al 2003). Ximelagatran was also evaluated for the prevention of stroke and systemic embolism in patients with AF in the SPORTIF III and V trials (Albers et al 2005; Olsson 2003). Based on the results of phase III trials, ximelagatran was launched in Europe in 2004 for the prevention of VTE after major orthopaedic surgery. However, it was withdrawn in 2006 due to concerns regarding liver toxicity and rebound cardiovascular effects. In the orthopedic development program, cardiovascular events and total mortality were significantly increased in the ximelagatran group compared with the control groups. Because of liver toxicity concerns, the US Food and Drug Administration never approved ximelagatran. 
FXa is another rational target for the development of antithrombotics. FXa promotes both coagulation and inflammation, and is at the point where the intrinsic and extrinsic coagulation cascade pathways meet. Inhibition of FXa is potentially more effective than targeting downstream thrombin, because the amount of activated coagulation factor generated from its inactive precursor increases at each level of the cascade. FXa is the primary site of amplification in the coagulation cascade: one molecule of FXa can facilitate the generation of more than 1000 thrombin molecules (Mann et al 2003). Proof of principal for pure FXa inhibition was provided by fondaparinux, which selectively but indirectly inhibits FXa by binding to antithrombin and potentiating its inhibition of FXa. Razaxaban (Bristol-Myers Squib) was one of the first direct FXa inhibitors developed. The antithrombotic potential of razaxaban was investigated in a phase II VTE prevention study after TKR (Lassen et al 2003). Four doses of razaxaban (range 25-100 mg given twice daily [bid]) were evaluated. The study showed a highly significant reduction of thromboembolic events with increased doses of razaxaban. However, the three higher dose arms of the study were stopped prematurely because of increased rates of major bleeding. Further development of razaxaban was halted and was replaced by development of another FXa inhibitor, apixaban.

There are several promising oral anticoagulants currently in clinical development, including the DTI dabigatran etexilate and the direct FXa inhibitors rivaroxaban and apixaban. This review will provide a critical appraisal of the clinical potential of these agents.

\section{Dabigatran}

Dabigatran (Boehringer Ingelheim) is a specific, competitive, and reversible DTI that is administered as the oral prodrug dabigatran etexilate (Eriksson et al 2004; Stangier et al 2005).
Dabigatran is formed by the rapid esterase-catalyzed conversion of dabigatran etexilate via two intermediary prodrugs (Blech et al 2008). Dabigatran binds to the active site of thrombin $\left(\mathrm{K}_{\mathrm{i}} 4.5 \mathrm{nM}\right)$ by hydrophobic interaction (Hauel et al 2002), thereby inhibiting the cleavage of fibrinogen to fibrin, and blocking the final step of the coagulation cascade, and thus thrombus formation. Dabigatran inhibits both free and fibrin-bound thrombin (Stangier et al 2007). The prodrug dabigatran etexilate is absorbed rapidly, but has low oral bioavailability (approximately 6.5\%) (Stangier et al 2007). Peak plasma concentrations of dabigatran occur approximately 2 hours after administration, and steady-state conditions are reached within 3 days after multiple dosing. The average terminal elimination half-life of dabigatran is 15 hours, protein binding is moderate $(25 \%-30 \%)$, and the compound is cleared predominantly via the renal pathway (Stangier et al 2007) (Table 2).

The antithrombotic potential of dabigatran for VTE prevention following THR or TKR was investigated in a double-blind, randomized, phase II dose-ranging study, BISTRO II (Eriksson et al 2005a). The primary efficacy outcome was the incidence of VTE (symptomatic, or asymptomatic, detected by venography) during 6-10 days of study drug. Of 1464 patients evaluable for the efficacy analysis, VTE occurred in 28.5\%, 17.4\%, 13.1\%, 16.6\%, and $24.0 \%$ of patients receiving dabigatran etexilate 50 , $150,225 \mathrm{mg}$ bid, or $300 \mathrm{mg}$ once daily (od), and enoxaparin $40 \mathrm{mg}$ od, respectively. A significant dose-dependent decrease in VTE occurred with increasing doses of dabigatran etexilate $(\mathrm{p}<0.0001)$. Major bleeding was low with $50 \mathrm{mg}$ bid dabigatran etexilate, relative to enoxaparin $(0.3 \%$ and $2.0 \%$, respectively; $\mathrm{p}=0.047$ ), but was elevated relative to enoxaparin at higher daily doses (Table 3 ).

Based on the results of BISTRO II, dabigatran $(220 \mathrm{mg}$ or $150 \mathrm{mg}$ od) was compared with enoxaparin $40 \mathrm{mg}$ od,

Table 2 Therapeutic target and pharmacologic properties of oral antithrombotic agents in clinical development

\begin{tabular}{|c|c|c|c|c|c|c|c|}
\hline Drug & Manufacturer & $\begin{array}{l}\text { Molecular } \\
\text { weight }\end{array}$ & $\begin{array}{l}\text { Therapeutic } \\
\text { target }\end{array}$ & Half-life & $\begin{array}{l}\text { Time } \\
\text { to } C_{\max }\end{array}$ & Bioavailability & $\begin{array}{l}\text { Mode } \\
\text { of elimination }\end{array}$ \\
\hline Dabigatran & Boehringer Ingelheim & $\begin{array}{l}628 \text { (dabigatran } \\
\text { etexilate), 47I } \\
\text { (dabigatran) }\end{array}$ & $\begin{array}{l}\text { Thrombin } \\
\text { (Factor Ila) }\end{array}$ & $\begin{array}{l}\text { I4-17 h with multiple } \\
\text { doses; 7-9 h with single } \\
\text { doses }\end{array}$ & $2 \mathrm{~h}$ & $\begin{array}{l}\text { Approximately } \\
6.5 \% \text { in humans }\end{array}$ & Renal \\
\hline Rivaroxaban & $\begin{array}{l}\text { Bayer HealthCare AG } \\
\text { and Johnson \& Johnson } \\
\text { Pharmaceutical Research and } \\
\text { Development, L.L.C. }\end{array}$ & 436 & Factor $\mathrm{Xa}$ & $\begin{array}{l}\text { Approximately } 9 \mathrm{~h} \\
\text { in healthy subjects; } \\
\text { approximately } 12 \mathrm{~h} \text { in } \\
\text { elderly subjects ( }>75 \mathrm{yr})\end{array}$ & $2-4 h$ & $\begin{array}{l}\text { Approximately } \\
80 \% \text { in humans }\end{array}$ & $\begin{array}{l}\text { Renal/biliary- } \\
\text { fecal }\end{array}$ \\
\hline Apixaban & $\begin{array}{l}\text { Bristol-Myers Squibb and } \\
\text { Pfizer }\end{array}$ & 460 & Factor $\mathrm{Xa}$ & $\begin{array}{l}\text { Approximately } 12 \mathrm{~h} \text {; } \\
\text { terminal half-life } 8-15 \mathrm{~h}\end{array}$ & $3 \mathrm{~h}$ & $\begin{array}{l}\text { Chimps (5I\%); } \\
\text { dogs }(88 \%) \text {; } \\
\text { rats }(43 \%)\end{array}$ & Renal/fecal \\
\hline
\end{tabular}


for VTE prevention for 35 days in patients after THR in the phase III RE-NOVATE study (Eriksson et al 2007a). In this study, the primary endpoint of non-inferiority to enoxaparin was met; the primary outcome (deep vein thrombosis [DVT], pulmonary embolism [PE], and all-cause mortality) occurred in $8.6 \%$ and $6.0 \%$ of patients receiving 150 and $220 \mathrm{mg}$ oral dabigatran etexilate od, respectively, compared with $6.7 \%$ of patients receiving enoxaparin. The rate of major bleeding was $1.3 \%$ and $2.0 \%$ in the 150 and $220 \mathrm{mg}$ od dabigatran etexilate arms, respectively, compared with $1.6 \%$ in the enoxaparin group (Table 3 ).

The efficacy and safety of dabigatran for VTE prevention after TKR was evaluated in two phase III studies: RE-MODEL (Eriksson et al 2006c) and RE-MOBILIZE (The RE-MOBILIZE Writing Committee 2008). In the RE-MODEL study, 2183 patients were randomized to receive dabigatran etexilate 150 or $220 \mathrm{mg}$ od, or enoxaparin $40 \mathrm{mg}$ od for 6-10 days. The primary efficacy outcome (a composite of total VTE and mortality) occurred in $37.7 \%$ of the enoxaparin group compared with $36.4 \%$ and $40.5 \%$ of the dabigatran 220 and $150 \mathrm{mg}$ groups, respectively. The incidence of major bleeding was similar between the three groups. Overall, both doses of dabigatran were non-inferior to enoxaparin, with a similar safety profile. However, in the RE-MOBILIZE study, non-inferiority of dabigatran to enoxaparin was not demonstrated. In this study, 2596 patients were randomized to either dabigatran 150 or $220 \mathrm{mg}$ od or enoxaparin $30 \mathrm{mg}$ bid for $12-15$ days. The incidence of the primary outcome was $33.7 \%, 31.1 \%$ and $25.3 \%$, respectively. The largest component of the primary outcome, distal DVT, occurred in $30.5 \%$ of patients receiving dabigatran $150 \mathrm{mg}$ od, $27.6 \%$ of patients receiving dabigatran $220 \mathrm{mg}$ od, and $23.0 \%$ of patients receiving enoxaparin. The incidence of major bleeding events was $0.6 \%$ for both dabigatran 150 and $220 \mathrm{mg}$ and $1.4 \%$ for enoxaparin (Table 3).

In a pooled analysis of the RE-MODEL, RE-MOBILIZE, and RE-NOVATE studies (Caprini et al 2007), major VTE and VTE-related death occurred in $3.3 \%$ of the enoxaparin group versus $3.0 \%$ of the dabigatran etexilate $220 \mathrm{mg}$ group and $3.8 \%$ of the dabigatran etexilate $150 \mathrm{mg}$ group. Major bleeding events were infrequent, and occurred at similar rates across all groups: enoxaparin 1.4\%, dabigatran etexilate $220 \mathrm{mg} \mathrm{1.4 \%}$, and dabigatran etexilate $150 \mathrm{mg} \mathrm{1.1 \%}$.

In summary, dabigatran has demonstrated non-inferiority and a similar safety profile to enoxaparin for VTE prevention after THR, and represents a viable, orally administered alternative to enoxaparin in this setting. The results for VTE prevention after TKR are less conclusive. Dabigatran demonstrated non-inferiority to enoxaparin in one phase III study but not in another, although it should be noted that different enoxaparin dosing regimens were used in each of these studies; bleeding rates with dabigatran were similar to enoxaparin in both studies. Based on the results of phase III studies, dabigatran has recently been approved in the European Union for the prevention of VTE following major orthopaedic surgery in adults.

Dabigatran is currently being investigated in three further phase III trials: RE-LY, a study comparing the efficacy and safety of dabigatran with warfarin for the prevention of stroke and systemic embolism in patients with non-valvular AF; RE-COVER, a randomized study comparing the efficacy and safety of dabigatran etexilate with warfarin for the treatment of acute symptomatic VTE, following initial treatment with a parenteral anticoagulant; and RE-MEDY, a randomized, active controlled study to evaluate the efficacy and safety of oral dabigatran etexilate compared with warfarin, for the secondary prevention of VTE.

\section{Rivaroxaban}

Rivaroxaban (Bayer HealthCare AG and Johnson \& Johnson Pharmaceutical Research and Development, L.L.C.) is a once-daily, oral, direct FXa inhibitor. It selectively and competitively binds to $\mathrm{FXa}\left(\mathrm{K}_{\mathrm{i}} 0.4 \mathrm{nM}\right)$ with $1: 1$ stoichiometry, blocking the interaction of FXa with its substrate prothrombin (Hirsh et al 2005). Rivaroxaban binds to the active site of FXa, its chlorothiophene moiety directed into the $\mathrm{S} 1$ pocket, and does not require highly basic groups like amidines for FXa affinity (Roehrig et al 2005). Binding inhibits not only free FXa but also fibrin-bound FXa and prothrombinase activity (Graff et al 2007). Rivaroxaban has high bioavailability and a dual mode of elimination, with one-third of the dose excreted unchanged via the kidneys, and two-thirds metabolized by the liver (Weinz et al 2004). Maximum plasma levels of rivaroxaban occur 2-4 hours after oral administration and elimination of rivaroxaban from plasma occurs with a terminal half-life of 5-9 hours in young individuals, and 11-12 hours in the elderly (Kubitza et al 2005b; Kubitza et al 2007c) (Table 2).

Three phase IIb trials, ODIXa-HIP2 (Eriksson et al 2006a), ODIXa-KNEE (Turpie et al 2005), and ODIXa-OD-HIP (Eriksson et al 2006b), were initiated to investigate the antithrombotic potential of rivaroxaban for VTE prevention following major orthopaedic surgery (THR or TKR). The primary efficacy outcome in these trials was the composite of any DVT, non-fatal PE, and all-cause mortality, and the primary safety outcome was major, post-operative bleeding. 


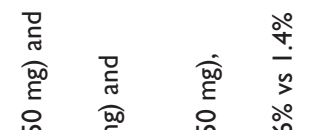

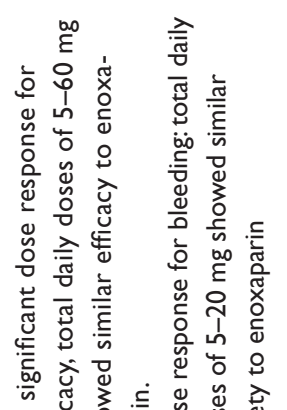

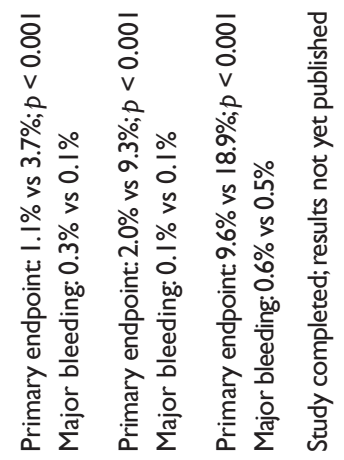

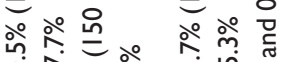

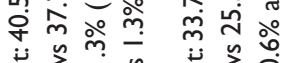

:

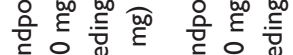

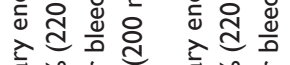

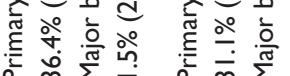

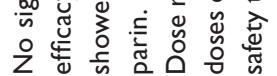

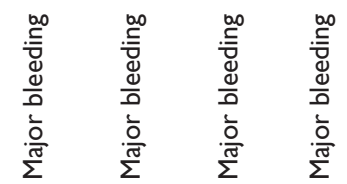

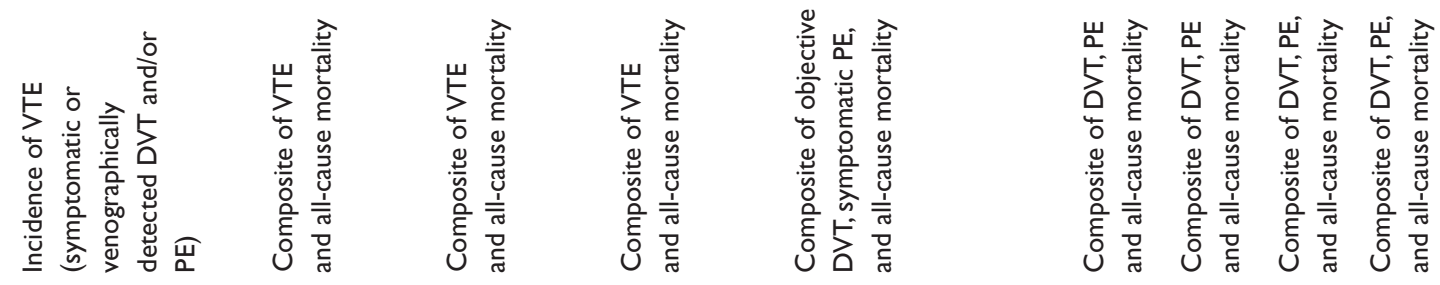

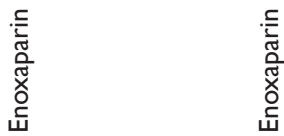

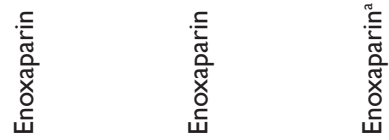

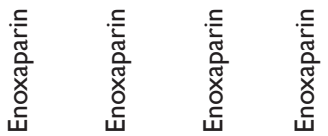

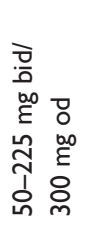

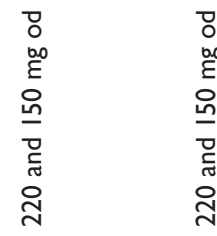

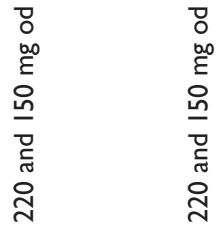

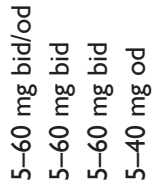

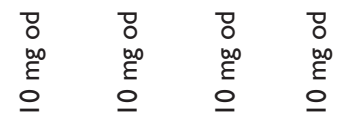

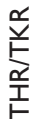

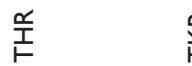

$\stackrel{\sim}{\stackrel{v}{\vdash}}$

$\stackrel{\propto}{\stackrel{ }{F}}$

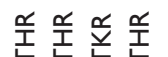

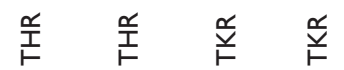

$=$

$\equiv$

$\equiv$

$\equiv$

$===$

$\equiv \equiv \equiv \equiv$

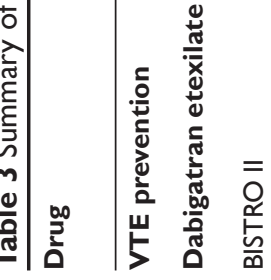

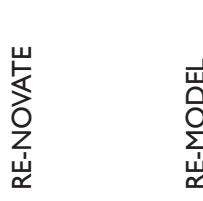

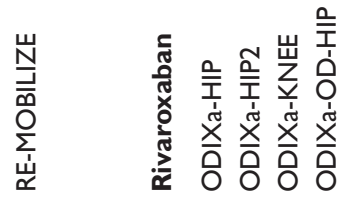

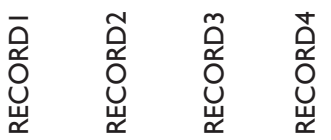




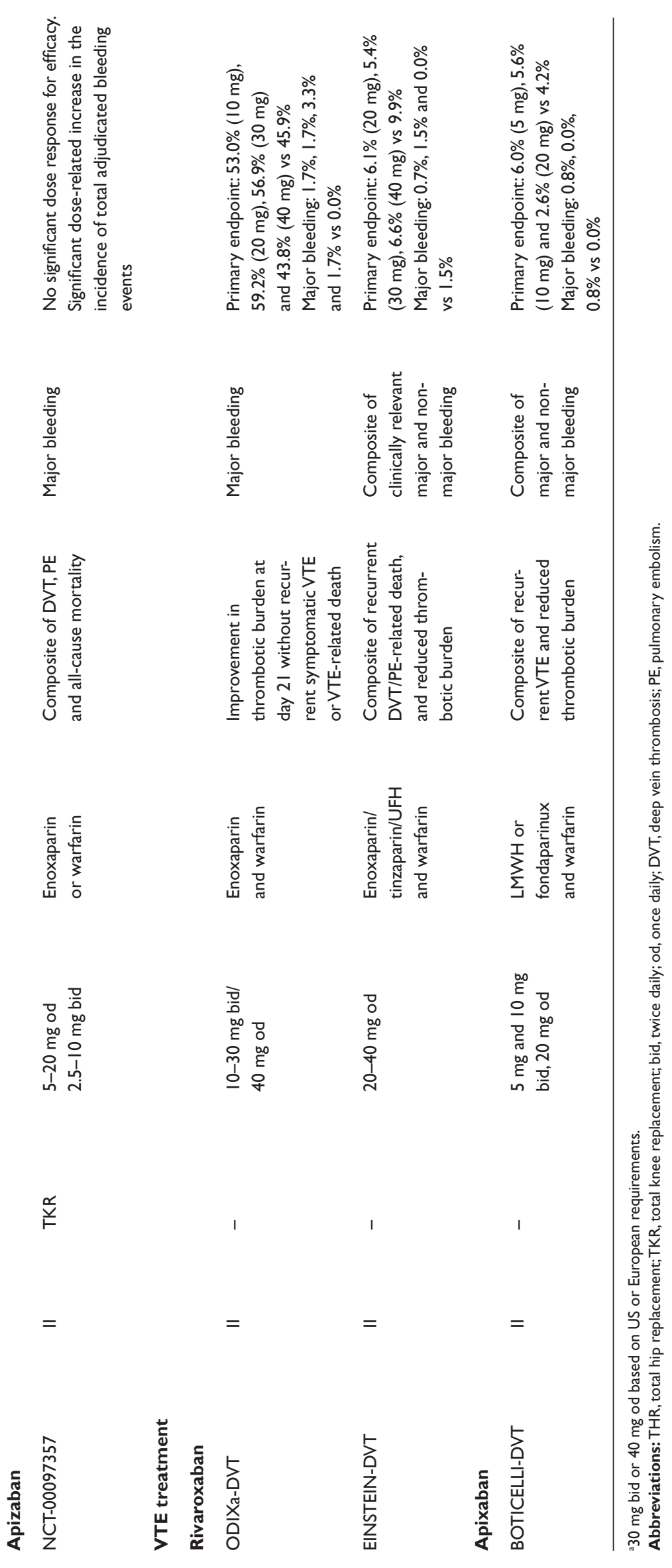


These trials were designed to allow pooling of the results and had the same independent blinded adjudication committees. Subjects were randomized to receive various doses of oral rivaroxaban (administered bid in two studies and od in one study) or subcutaneous enoxaparin (30 or $40 \mathrm{mg}$ ) for 5-9 days after surgery. The results of the phase II bid studies showed that total daily doses of 5-20 mg rivaroxaban warranted further investigation, while the od study demonstrated that a $10 \mathrm{mg}$ once-daily dose of rivaroxaban provided the optimum balance between efficacy and safety. Based on these findings, a once-daily $10 \mathrm{mg}$ dose of rivaroxaban was evaluated in phase III studies (Table 3).

The RECORD1 trial compared extended prophylaxis with rivaroxaban with extended enoxaparin after THR (Eriksson et al 2008). Patients received either oral rivaroxaban (10 mg od), started 6-8 hours after surgery for $35 \pm 4$ days, or subcutaneous enoxaparin (40 mg od), started the evening before surgery. In this study, the criteria for non-inferiority of rivaroxaban vs enoxaparin were met and testing for superiority was performed. The primary efficacy outcome (the composite of DVT, PE, and all-cause mortality) occurred in $18 / 1595(1.1 \%)$ of patients treated with rivaroxaban compared with $58 / 1558(3.7 \%)$ of those receiving enoxaparin ( $\mathrm{p}<0.001)$, demonstrating a relative risk reduction $(\mathrm{RRR})$ of $70 \%$. The incidence of major bleeding was similar in both groups (6/2209 [0.3\%] with rivaroxaban vs 2/2224 [0.1\%] with enoxaparin) (Table 3 ).

In RECORD2, extended prophylaxis with rivaroxaban was compared with short-term enoxaparin followed by placebo for prevention of VTE after THR in 2509 patients (Kakkar et al 2008). Patients received subcutaneous enoxaparin $40 \mathrm{mg}$ od, beginning the evening before surgery, continuing for 10-14 days (short-term prophylaxis), and followed by placebo until day $35 \pm 4$, or oral rivaroxaban $10 \mathrm{mg}$ od beginning 6-8 hours after surgery and continuing for $35 \pm 4$ days (extended prophylaxis). The primary efficacy outcome (composite of DVT, PE, and all-cause mortality) occurred in $17 / 864(2.0 \%)$ of patients given extended prophylaxis with rivaroxaban compared with $81 / 869(9.3 \%)$ of patients given short-term prophylaxis with enoxaparin ( $p<0.0001)$, demonstrating an RRR of $79 \%$. The rate of major bleeding was low and similar in those receiving extended prophylaxis with rivaroxaban and short-term enoxaparin (1/1228 [0.1\%] vs $1 / 1229$ [0.1\%], respectively) (Table 3).

The RECORD3 trial evaluated oral rivaroxaban (10 mg od) compared with subcutaneous enoxaparin (40 mg od) for the prevention of VTE after TKR in 2531 patients (Lassen et al 2008). The primary efficacy outcome (the composite of DVT, PE, and all-cause mortality) occurred in 79/824 $(9.6 \%)$ of patients receiving rivaroxaban compared with $166 / 878(18.9 \%)$ of those receiving enoxaparin $(p<0.001)$, demonstrating an RRR of 49\%. Major bleeding occurred in $7 / 1220(0.6 \%)$ administered rivaroxaban and 6/1239 (0.5\%) of patients administered enoxaparin $(\mathrm{p}=0.77)($ Table 3$)$.

RECORD4 compared once-daily oral rivaroxaban (10 mg) with twice-daily subcutaneous enoxaparin (30 mg) for VTE prophylaxis after TKR in 3148 randomized patients (Turpie et al 2008). The primary efficacy outcome was the same as for RECORD3 and occurred in significantly fewer patients in the rivaroxaban group. The rate of major bleeding was similar in the rivaroxaban and enoxaparin groups.

Rivaroxaban was also evaluated for VTE treatment in the phase II EINSTEIN-DVT (Buller et al 2008b) and ODIXa-DVT (Agnelli et al 2007a) trials. In these doseranging studies, both od and bid rivaroxaban dosing had similar efficacy to standard enoxaparin. In addition, a low rate of bleeding was observed with all rivaroxaban doses, suggesting that long-term treatment with rivaroxaban could be possible (Table 3 ). In the ODIXa-DVT study, the doses of rivaroxaban selected for evaluation (20-60 mg total daily doses) were based on pharmacokinetic and pharmacodynamic analyses, as well as results of VTE prevention trials in which a $10 \mathrm{mg}$ od dose appeared to be optimal - for treatment of established thrombosis, a minimum of two times the prophylactic dose was considered appropriate. In combination with results of the EINSTEIN-DVT study, where $20-40 \mathrm{mg}$ od doses of rivaroxaban were evaluated, the lowest ( $20 \mathrm{mg} /$ day) dose of rivaroxaban was chosen for evaluation in phase III clinical trials.

In summary, extended prophylaxis with rivaroxaban not only demonstrated non-inferiority, but was significantly more effective than both extended prophylaxis and short-term prophylaxis with enoxaparin after THR. Rivaroxaban was also superior to enoxaparin for the prevention of VTE after TKR. Bleeding rates with rivaroxaban were similar to enoxaparin in each of the three studies, even in the RECORD2 study where extended prophylaxis with rivaroxaban was compared with short-term prophylaxis with enoxaparin. Based on these promising results, rivaroxaban represents a viable, oral alternative to enoxaparin for prevention of VTE following major orthopaedic surgery.

Other phase III trials with rivaroxaban are currently underway. Rivaroxaban is being evaluated for VTE treatment in a phase III study of patients with acute symptomatic DVT (EINSTEIN-DVT evaluation) or acute symptomatic PE (EINSTEIN-PE evaluation), and for long-term prevention 
of recurrent symptomatic VTE in patients with symptomatic DVT or PE (EINSTEIN-extension study). A phase III study of rivaroxaban for VTE prophylaxis in medically ill patients has also been initiated (MEGELLAN), and rivaroxaban is being compared with warfarin for stroke prevention in patients with AF (ROCKET AF). Finally, rivaroxaban in combination with aspirin alone or with aspirin and a thienopyridine is being investigated in a phase II study of subjects with acute coronary syndromes (ATLAS ACS TIMI 46 Trial).

\section{Apixaban}

Apixaban (Bristol-Myers Squib), a follow-up compound to razaxaban, is a selective, reversible, direct FXa inhibitor. Apixaban has a $\mathrm{K}_{\mathrm{i}}$ for $\mathrm{FXa}$ of $0.8 \mathrm{nM}$, and it inhibits prothrombinase activity as well as free FXa (Weitz 2006). Apixaban demonstrates relatively high oral bioavailability in animal models (51\% in chimps; $88 \%$ in dogs; $43 \%$ in rats) (He et al 2006) and has a half-life of approximately 12 hours in humans (Frost et al 2007). Maximum plasma levels of apixaban are reached approximately 3 hours after administration. Apixaban is cleared through renal (approximately 25\%) and fecal routes (approximately 70\%) (Lassen et al 2006) (Table 2).

The antithrombotic potential of apixaban, given od or bid, was investigated in a phase II trial in patients who had undergone TKR (Lassen et al 2007). The incidence of the primary efficacy outcome (composite of VTE and all-cause mortality during administration) decreased with increasing apixaban dose versus comparators (enoxaparin $30 \mathrm{mg}$ bid or warfarin [international normalized ratio (INR) 1.8-3.0]), although the trend was not significant ( $p=0.09$ with od/bid apixaban regimens combined, $\mathrm{p}=0.19$ for od and $\mathrm{p}=0.13$ for bid dosing). Overall, total VTE rates were slightly lower in the bid than in the od apixaban arms. A significant dose-related increase in the incidence of total adjudicated bleeding events was noted in the od $(\mathrm{p}=0.01)$ and bid $(\mathrm{p}=0.02)$ apixaban groups; there was no difference between od and bid regimens. Because, at each total dose of apixaban, there were lower point estimates for the primary outcome with bid versus od dosing, bid dosing was established as the preferred regimen to be tested in a comprehensive phase III program. Apixaban was also evaluated for VTE treatment in the phase II BOTICELLI trial (Buller et al 2008a). The primary efficacy outcome was the composite of symptomatic recurrent VTE and deterioration of the thrombotic burden. The primary safety outcome was the composite of major and clinically relevant non-major bleeding. Primary efficacy outcome rates were $6.0 \%$ for patients in the $5 \mathrm{mg}$ bid apixaban group,
$5.6 \%$ for patients in the $10 \mathrm{mg}$ bid group, and $2.6 \%$ in the $20 \mathrm{mg}$ od group compared with $4.2 \%$ for the control group (LMWH or fondaparinux followed by VKA). Rates of major bleeding were $0.8 \%$ (5 mg bid), $0.0 \%$ (10 mg bid), $1.6 \%$ $20 \mathrm{mg}$ od), and $0.0 \%$ (control) (Table 3).

Apixaban is currently being evaluated in phase III VTE prevention studies following TKR (ADVANCE-1, $2.5 \mathrm{mg}$ oral apixaban bid vs $30 \mathrm{mg}$ subcutaneous enoxaparin bid; ADVANCE-2, $2.5 \mathrm{mg}$ oral apixaban bid vs $40 \mathrm{mg}$ subcutaneous enoxaparin od), THR (ADVANCE-3, $2.5 \mathrm{mg}$ oral apixaban bid vs $40 \mathrm{mg}$ subcutaneous enoxaparin od), and in acutely medically ill patients. Apixaban is also being compared with acetylsalicylic acid (ASA) in a phase III study for stroke prevention in AF (AVERROES) and with warfarin for prevention of stroke and systemic embolism in subjects with non-valvular AF (ARISTOTLE). A phase II, placebo-controlled, dose-ranging study to evaluate the safety and efficacy of apixaban in patients with a recent ACS is also ongoing.

In summary, although apixaban is at an earlier stage of development than either dabigatran or rivaroxaban, it has demonstrated promising safety and efficacy compared with the standard of care in phase II clinical trials for VTE prevention and treatment. However, based on the phase II dose-finding studies, bid rather than od apixaban dosing has been selected for further investigation in phase III VTE prevention trials. Dabigatran and rivaroxaban by comparison are administered od in this indication.

\section{Other oral antithrombotics in clinical development}

Numerous other oral antithrombotic agents that directly target FXa are currently in early clinical development (phase I or phase II). Betrixaban (Portola) is a compound with a $\mathrm{K}_{\mathrm{i}}$ for FXa of $0.117 \mathrm{nM}$, bioavailability of $47 \%$, and a half-life of 19 hours. In animal models, betrixaban has demonstrated antithrombotic activity (Abe et al 2006) and, in a phase I dose-escalation study in 64 subjects, betrixaban displayed a long half-life, suggesting od dosing may be feasible. A phase II study to evaluate the efficacy and safety of betrixaban for prevention of VTE is underway.

The compound DU-176b (Daiichi Sankyo) has a Ki for FXa of $0.56 \mathrm{nM}$ and a 10,000-fold higher selectivity for FXa than for thrombin (Morishima et al 2004). DU-176b has also demonstrated promising antithrombotic potential in both venous and arterial models of thrombosis in rats (Eriksson et al 2005b). In a phase I study in healthy subjects $(\mathrm{n}=12)$, DU-176b demonstrated a significant reduction in 
thrombus formation at both venous and arterial rheologies, up to 5 hours post-dose (Zafar et al 2007). Phase IIb studies of DU-176b in VTE prevention, stroke prevention in patients with AF, and in patients with ACS are planned or have been initiated.

YM150 (Astellas) is a compound that has a Ki for FXa of $31 \mathrm{nM}$, and inhibits activation of prothrombin induced by prothrombinase, free FXa, and whole-blood clots (Iwatsuki et al 2006). Proof of concept was demonstrated in a phase IIa dose-escalation study to assess the efficacy and safety of YM150 for VTE prevention after THR (Eriksson et al 2007b). Patients $(\mathrm{n}=174)$ undergoing hip replacement surgery were randomized to receive oral od YM150 (3-60 mg) or enoxaparin $40 \mathrm{mg}$ od for 7-10 days. The primary outcome (major and/or clinically relevant nonmajor bleeding) occurred in $2.9 \%$ and $5.7 \%$ of the 3 and 10 mg YM150 dose groups, respectively. Of 147 patients with an evaluable venogram (84\%), VTE (DVT/PE) occurred in $51.9 \%, 38.7 \%, 22.6 \%$, and $18.5 \%$ of patients in the 3, 10, 30, and $60 \mathrm{mg}$ YM150 dose groups, respectively. A significant YM150 dose-related trend in VTE incidence was demonstrated $(\mathrm{p}=0.006)$. VTE occurred in $38.7 \%$ of patients receiving enoxaparin.

LY-517717 (Eli Lilly) is an FXa inhibitor with 1000-fold greater selectivity for FXa than related serine proteases. In preclinical studies, LY-517717 was shown to have a $\mathrm{K}_{\mathrm{i}}$ of 4.6 to $6.6 \mathrm{nM}$ and an oral bioavailability of 25\%-82\% (Turpie 2007). LY-517717 has a half-life of approximately 25 hours in humans, potentially making it suitable for od dosing. In a phase II, non-inferiority study, LY-517717 has been compared with enoxaparin for VTE prevention in patients $(\mathrm{n}=511)$ undergoing THR or TKR. Participants were randomized to receive one of six od doses of LY-517717 (starting 6-8 hours after wound closure) or od enoxaparin $40 \mathrm{mg}$ (starting the evening before surgery). The primary efficacy endpoint was DVT on mandatory bilateral venography within 12 hours of the last dose of study drug or objectively confirmed symptomatic VTE before day 30. Administration of LY-517717 resulted in a dose-dependent decrease in the incidence of thromboembolic events $(p=0.0001)$. The incidences of VTE were $19 \%, 19 \%$, and $16 \%$ with 100 , 125 , and $150 \mathrm{mg}$ of LY -517717 , respectively, compared with 21\% with enoxaparin. These doses were non-inferior to enoxaparin with respect to the primary efficacy endpoint. Bleeding events were similar in all study arms (100 mg: 0.9\%; $125 \mathrm{mg}$ : 0.0\%; $150 \mathrm{mg}$ : 1.0\%; and enoxaparin: $2.2 \%$ ) (Agnelli et al 2007b).
A further oral, direct FXa inhibitor, PD 0348292 (Pfizer), is currently being evaluated for efficacy and safety in the prevention of VTE following elective TKR in a phase IIb randomized, multicenter, dose-ranging study.

\section{Patients' perspectives}

Patient non-compliance to anticoagulation therapy is common in clinical practice (McBride et al 2007). Patients receiving VKAs, eg, warfarin, require frequent monitoring and dose adjustments to achieve the desired therapeutic range (target INR usually $2-3$ ). If the INR is out of the target range, bleeding and other adverse events may occur (Ansell et al 2001; Gurwitz et al 2007). The multiple food and drug interactions associated with VKAs also mean patients have to endure dietary restrictions, and discontinuation of other medications may be necessary for effective anticoagulation to be achieved. A combination of these factors can potentially result in patients stopping their therapy, or becoming anxious about potential side-effects. Frequent dose adjustments or complex dosing regimens can be confusing (Hixson-Wallace et al 2001), particularly for elderly patients, where non-compliance can be a particular problem (Gurwitz et al 1992).

Patients receiving chronic warfarin therapy who have poor anticoagulation control (usually expressed as time in range) are at increased risk for adverse events (Oake et al 2007). In a recent, well-controlled clinical trial (ACTIVE-W), in which oral anticoagulation with a VKA was shown to be superior to the combination therapy of ASA and clopidogrel for prevention of vascular events in patients with AF, patients receiving oral anticoagulation therapy had INR values in the therapeutic range (2-3) $63.8 \%$ of the time; below 2, $20.8 \%$ of the time; and above $3,15.4 \%$ of the time (Connolly et al 2006). The quality of warfarin anticoagulation control is potentially associated with patient compliance, demographics, knowledge about therapy, and perceived impact on quality of life. In a study of 52 patients from two anticoagulation clinics in the US (Davis et al 2005), only 14\% of patients demonstrated good anticoagulation control. Adequate compliance was reported by $50 \%$ of patients and was significantly associated with good anticoagulation control $(\mathrm{p}=0.01)$. Good knowledge of anticoagulation therapy was reported in $37 \%$ of the group and $19 \%$ reported that warfarin negatively impacted their quality of life; however, these factors were not significantly associated with anticoagulation control. These findings highlight the importance of patient compliance for achieving good coagulation control with VKAs. However, the situation in general practice may be worse than described in the above 
studies, because it has been shown that anticoagulation control in routine medical care is potentially of a lower quality than in controlled clinical trials or specialized anticoagulation clinics (Menzin et al 2005).

Although UFH, LMWHs, and fondaparinux are easier to manage than VKAs, they require parenteral administration, which is inconvenient for use outside the clinic. UFH has the added disadvantage of requiring coagulation monitoring and is also associated with HIT and osteoporosis. The requirement for monitoring during VKA and UFH therapy necessitates regular visits to the clinic and potential disruption to daily routine.

From a patient perspective, a preferred anticoagulant would have a convenient mode of administration and a high efficacy-to-safety index, with freedom from hemorrhagic or non-hemorrhagic (HIT, skin reactions or osteoporosis) side-effects. Other desirable attributes would include a predictable dose response that allows dosing without the need for laboratory monitoring, a rapid onset of action so that parenteral bridging therapy is not necessary, and minimal interaction with other drugs or food.

The future availability of the novel antithrombotics described in this article could provide patients with anticoagulants possessing many of these attributes. These anticoagulants are administered either once or twice daily in a convenient oral form and have a rapid onset of action. Because they directly target one specific factor in the coagulation cascade, their pharmacology is likely to be more predictable, negating the need for monitoring. Close relationships between phamacokinetic and pharmacodynamic measurements have been demonstrated for dabigatran and rivaroxaban. Plasma concentrations of dabigatran correlate well with activated partial thromboplastin time and ecarin clotting time (Stangier et al 2007), and rivaroxaban plasma concentrations show a close correlation with FXa activity and prothrombin time. These findings highlight the predictable pharmacology of dabigatran and rivaroxaban compared with the VKAs (Kubitza et al 2005a). In addition, it has been demonstrated that dabigatran and rivaroxaban have no clinically relevant interaction with food (Kubitza et al 2006; Stangier et al 2007), and a low propensity for drug-drug interactions (Blech et al 2008; Kubitza et al 2006; Kubitza et al 2007a; Kubitza et al 2007b), although concomitant use of dabigatran with ASA significantly increases the risk of bleeding compared with dabigatran alone (Ezekowitz et al 2007). Drug-drug interactions and the effect of food on apixaban have not currently been reported. Phase III clinical trials of dabigatran and rivaroxaban for the prevention of VTE have also demonstrated that non-hemorrhagic side-effects are rare, and that the risk of bleeding is similar compared with enoxaparin (Eriksson et al 2007a; Eriksson et al 2008; Kakkar et al 2008; Lassen et al 2008; The RE-MOBILIZE Writing Committee 2008). Rivaroxaban and dabigatran are currently being evaluated in phase III trials for VTE treatment, secondary VTE prevention, prevention of stroke in AF (rivaroxaban), and prevention of stroke and systemic embolism in non-valvular AF (dabigatran). Phase III trials for the prevention of VTE, the prevention of stroke in AF, and the prevention of stroke and systemic embolism in non-valvular $\mathrm{AF}$ are ongoing for apixaban.

\section{Conclusions}

Despite their unpredictable pharmacologic profile and associated risks, VKAs are still widely used anticoagulants. They can be administered orally, usually reducing the length of hospital stay. Although if managed well VKAs are highly effective, the need for frequent monitoring of the INR has a negative impact on their cost-effectiveness. In addition, noncompliance with VKA therapy results in many patients not receiving optimal anticoagulation and increases the risk of uncontrolled bleeding. UFH, LMWHs and fondaparinux are much safer and easier to manage than VKAs but they require parenteral administration, making them less convenient for use outside the hospital. There is a significant unmet need for a convenient, predictable anticoagulant that is both effective and safe for the prevention and treatment of thromboembolic disorders. Several novel oral anticoagulants have recently demonstrated efficacy and safety at least equivalent to standard treatments in randomized phase III trials and are now in the advanced stages of clinical development. The predictable pharmacologic profile and anticoagulant effect of these agents removes the need for monitoring, and the associated hospital costs and inconvenience to the patient. In addition, oral dosing means patients can receive anticoagulation therapy at home.

The introduction of these orally active, novel anticoagulants is likely to result in an improvement in the prevention and treatment of thromboembolic disorders, and may overcome many of the concerns associated with currently available therapies. Because of their predictable pharmacology, these newer agents are also reliable and may be safer than established antithrombotic drugs.

\section{Acknowledgments}

The authors would like to acknowledge Sarah Atkinson of Chameleon Communications International, who 
provided medical writing services with funding from BayerHealthcare AG.

\section{Disclosures}

The authors have conflicts of interest to disclose.

\section{Abbreviations}

ACCP, American College of Chest Physicians; AF, atrial fibrillation; aPTT, activated partial thromboplastin time; ASA, acetylsalicylic acid; bid, twice daily; AT, antithrombin; DTI, direct thrombin inhibitor; DVT, deep vein thrombosis; HIT, heparin-induced thrombocytopenia; INR, international normalized ratio; LMWH, low-molecular-weight heparin; PE, pulmonary embolism; TF, tissue factor; TKR, total knee replacement; THR, total hip replacement; od, once daily; UFH, unfractionated heparin; VKA, vitamin K antagonist; VTE, venous thromboembolism.

\section{References}

Abe K, Siu G, Edwards S, et al. 2006. Animal models of thrombosis help predict the human therapeutic concentration of PRT54021, a potent oral Factor Xa inhibitor. Blood, 108:Abstract 901.

Agnelli G, Gallus A, Goldhaber SZ, et al. 2007a. Treatment of proximal deep-vein thrombosis with the oral direct Factor Xa inhibitor rivaroxaban (BAY 59-7939): the ODIXa-DVT (oral direct Factor Xa inhibitor BAY 59-7939 in patients with acute symptomatic deep-vein thrombosis) study. Circulation, 116:180-7.

Agnelli G, Haas S, Ginsberg JS, et al. 2007b. A phase II study of the oral factor Xa inhibitor LY517717 for the prevention of venous thromboembolism after hip or knee replacement. J Thromb Haemost, 5:746-53.

Albers GW, Diener HC, Frison L, et al. 2005. Ximelagatran vs warfarin for stroke prevention in patients with nonvalvular atrial fibrillation: a randomized trial. JAMA, 293:690-8.

Ansell J. 2007. Factor Xa or thrombin: is factor Xa a better target? J Thromb Haemost, 5:60-4.

Ansell J, Hirsh J, Dalen J, et al. 2001. Managing oral anticoagulant therapy. Chest, 119:22S-38S.

Ansell J, Hirsh J, Poller L, et al. 2004. The pharmacology and management of the vitamin K antagonists: the Seventh ACCP Conference on Antithrombotic and Thrombolytic Therapy. Chest, 126:204S-33S.

Blech S, Ebner T, Ludwig-Schwellinger E, et al. 2008. The metabolism and disposition of the oral direct thrombin inhibitor, dabigatran, in humans. Drug Metab Dispos, 36:386-99.

Buller H, Deitchman D, Prins M, et al. 2008a. Efficacy and safety of the oral direct factor Xa inhibitor apixaban for symptomatic deep-vein thrombosis. The Botticelli DVT dose-ranging study. J Thromb Haemost. In press.

Buller HR, Agnelli G, Hull RD, et al. 2004. Antithrombotic therapy for venous thromboembolic disease: the Seventh ACCP Conference on Antithrombotic and Thrombolytic Therapy. Chest, 126:401S-28S.

Buller HR, Lensing AW, Prins MH, et al. 2008b. A dose-ranging study evaluating once-daily oral administration of the factor Xa inhibitor Rivaroxaban in the treatment of patients with acute symptomatic deep vein thrombosis. The EINSTEIN-DVT Dose-Ranging Study. Blood. In press.

Caprini JA, Hwang E, Hantel S, et al. 2007. The oral direct thrombin inhibitor, dabigatran etexilate, is effective and safe for prevention of major venous thromboembolism following major orthopaedic surgery. $J$ Thromb Haemost, 5:Abstract O-W-050.

Colwell CW, Jr., Berkowitz SD, Davidson BL, et al. 2003. Comparison of ximelagatran, an oral direct thrombin inhibitor, with enoxaparin for the prevention of venous thromboembolism following total hip replacement. A randomized, double-blind study. J Thromb Haemost, 1:2119-30.
Connolly S, Pogue J, Hart R, et al. 2006. Clopidogrel plus aspirin versus oral anticoagulation for atrial fibrillation in the Atrial fibrillation Clopidogrel Trial with Irbesartan for prevention of Vascular Events (ACTIVE W.): a randomised controlled trial. Lancet, 367:1903-12.

Dantas GC, Thompson BV, Manson JA, et al. 2004. Patients' perspectives on taking warfarin: qualitative study in family practice. BMC Fam Pract, 5:15-23.

Davie EW. 1995. Biochemical and molecular aspects of the coagulation cascade. Thromb Haemost 74:1-6.

Davis NJ, Billett HH, Cohen HW, et al. 2005. Impact of adherence, knowledge, and quality of life on anticoagulation control. Ann Pharmacother, 39:632-6.

Eriksson BI, Agnelli G, Cohen AT, et al. 2003a. The direct thrombin inhibitor melagatran followed by oral ximelagatran compared with enoxaparin for the prevention of venous thromboembolism after total hip or knee replacement: the EXPRESS study. J Thromb Haemost, 1:2490-6.

Eriksson BI, Agnelli G, Cohen AT, et al. 2003b. Direct thrombin inhibitor melagatran followed by oral ximelagatran in comparison with enoxaparin for prevention of venous thromboembolism after total hip or knee replacement. Thromb Haemost, 89:288-96.

Eriksson BI, Borris L, Dahl OE, et al. 2006a. Oral, direct Factor Xa inhibition with BAY 59-7939 for the prevention of venous thromboembolism after total hip replacement. $J$ Thromb Haemost, 4:121-8.

Eriksson BI, Borris LC, Dahl OE, et al. 2006b. A once-daily, oral, direct Factor Xa inhibitor, rivaroxaban (BAY 59-7939., for thromboprophylaxis after total hip replacement. Circulation, 114:2374-81.

Eriksson BI, Borris LC, Friedman RJ, et al. 2008. Rivaroxaban versus enoxaparin for thromboprophylaxis after hip arthroplasty. N Engl J Med, 358:2765-75.

Eriksson BI, Dahl OE, Ahnfelt L, et al. 2004. Dose escalating safety study of a new oral direct thrombin inhibitor, dabigatran etexilate, in patients undergoing total hip replacement: BISTRO I. J Thromb Haemost, 2:1573-80.

Eriksson BI, Dahl OE, Buller HR, et al. 2005a. A new oral direct thrombin inhibitor, dabigatran etexilate, compared with enoxaparin for prevention of thromboembolic events following total hip or knee replacement: the BISTRO II randomized trial. J Thromb Haemost, 3:103-11.

Eriksson BI, Dahl OE, Rosencher N, et al. 2007a. Dabigatran etexilate versus enoxaparin for prevention of venous thromboembolism after total hip replacement: a randomised, double-blind, non-inferiority trial. Lancet, 370:949-56.

Eriksson BI, Dahl OE, van Dijk CN, et al. 2006c. A new oral anticoagulant, dabigatran etexilate, is effective and safe in preventing venous thromboembolism after total knee replacement surgery (the RE-MODEL trial). Blood, 108:Abstract 573.

Eriksson BI, Turpie AG, Lassen MR, et al. 2007b. A dose escalation study of YM150, an oral direct factor Xa inhibitor, in the prevention of venous thromboembolism in elective primary hip replacement surgery. $J$ Thromb Haemost, 5:1660-5.

Eriksson BI, Turpie AGG, Lassen MR, et al. 2005b. YM150, an oral direct Factor Xa inhibitor, as prophylaxis for venous thromboembolism in patients with elective primary hip replacement surgery. A dose escalation study. Blood, 106:Abstract 1865.

Esmon CT, Esmon NL, Le Bonniec BF, et al. 1993. Protein C activation. Methods Enzymol, 222:359-85.

Ezekowitz MD, Reilly P, Nehmiz G, et al. 2007. Dabigatran with or without concomitant aspirin compared with warfarin alone in patients with nonvalvular atrial fibrillation (PETRO Study. Am J Cardiol, 100:1419-26.

Fiessinger JN, Huisman MV, Davidson BL, et al. 2005. Ximelagatran vs low-molecular-weight heparin and warfarin for the treatment of deep vein thrombosis: a randomized trial. JAMA, 293:681-9.

Francis CW, Berkowitz SD, Comp PC, et al. 2003. Comparison of ximelagatran with warfarin for the prevention of venous thromboembolism after total knee replacement. $N$ Engl $J$ Med, 349:1703-12. 
Frost C, Yu Z, Moore K. 2007. Apixaban, an oral direct Factor Xa inhibitor: multiple-dose safety, pharmokinetics and pharmaccodynamics in healthy subjects. $J$ Thromb Haemost, 5:P-M-664.

Geerts WH, Bergqvist D, Pineo GF, et al. 2008. Prevention of venous thromboembolism: American College of Chest Physicians evidencebased clinical practice guidelines (8th Ed). Chest, 133:381-453.

Graff J, von Hentig N, Misselwitz F, et al. 2007. Effects of the oral, direct Factor Xa inhibitor rivaroxaban on platelet-induced thrombin generation and prothrombinase activity. J Clin Pharmacol, 47:1398-407.

Gurwitz JH, Avorn J, Ross-Degnan D, et al. 1992. Aging and the anticoagulant response to warfarin therapy. Ann Intern Med, 116:901-4.

Gurwitz JH, Field TS, Radford MJ, et al. 2007. The safety of warfarin therapy in the nursing home setting. Am J Med, 120:539-44.

Gustafsson D, Nystrom J, Carlsson S, et al. 2001. The direct thrombin inhibitor melagatran and its oral prodrug $\mathrm{H} 376 / 95$ : intestinal absorption properties, biochemical and pharmacodynamic effects. Thromb Res, 101:171-81.

Hauel NH, Nar H, Priepke H, et al. 2002. Structure-based design of novel potent nonpeptide thrombin inhibitors. J Med Chem, 45:1757-66.

Hawkins D. 2004. Limitations of traditional anticoagulants. Pharmacotherapy, 24:62S-5S

He K, He B, Grace JE, et al. 2006. Preclinical pharmacokinetic and metabolism of apixaban, a potent and selective Factor Xa inhibitor. Blood, 108:Abstract 910.

Hirsh J, O'Donnell M, Eikelboom JW. 2007. Beyond unfractionated heparin and warfarin: current and future advances. Circulation, 116:552-60.

Hirsh J, Guyatt G, Albers GW, et al. 2004. The Seventh ACCP Conference on Antithrombotic and Thrombolytic Therapy: evidence-based guidelines. Chest, 126:172S-3S.

Hirsh J, O'Donnell M, Weitz JI. 2005. New anticoagulants. Blood, 105:453-63.

Hirsh J, Warkentin TE, Shaughnessy SG, et al. 2001. Heparin and low-molecular-weight heparin: mechanisms of action, pharmacokinetics, dosing, monitoring, efficacy, and safety. Chest, 119:64S-94S.

Hixson-Wallace JA, Dotson JB, Blakey, SA. 2001. Effect of regimen complexity on patient satisfaction and compliance with warfarin therapy. Clin Appl Thromb Hemost, 7:33-7.

Iwatsuki Y, Shigenaga T, Moritani Y, et al. 2006. Biochemical and pharmacological profiles of YM150, an oral direct Factor Xa inhibitor. Blood, 108:Abstract 911.

Kakkar AK, Brenner B, Dahl OE, et al. 2008. Extended duration rivaroxaban versus short-term enoxaparin for the prevention of venous thromboembolism after total hip arthroplasty: a double-blind, randomised controlled trial. Lancet, 372:31-9.

Kubitza D, Becka M, Mueck W, et al. 2007a. Rivaroxaban (BAY 19. - an oral, direct Factor Xa inhibitor - has no clinically relevant interaction with naproxen. Br J Clin Pharmacol, 63:469-76.

Kubitza D, Becka M, Mueck W et al. 2007b. Rivaroxaban-a novel, oral, direct Factor Xa inhibitor has no clinically relevant interaction with acetylsalicylic acid or naproxen. J Thromb Haemost, 5. In press.

Kubitza D, Becka M, Mueck W, et al. 2007c. The effect of age and gender on the pharmacology and safety of the oral, direct Factor Xa inhibitor. $J$ Thromb Haemost, 5 . In press.

Kubitza D, Becka M, Voith B, et al. 2005a. Safety, pharmacodynamics, and pharmacokinetics of single doses of BAY 59-7939, an oral, direct factor Xa inhibitor. Clin Pharmacol Ther, 78:412-21.

Kubitza D, Becka M, Wensing G, et al. 2005b. Safety, pharmacodynamics, and pharmacokinetics of BAY 59-7939 - an oral, direct Factor Xa inhibitor - after multiple dosing in healthy male subjects. Eur J Clin Pharmacol, 61:873-80.

Kubitza D, Becka M, Zuehlsdorf M, et al. 2006. Effect of food, an antacid, and the $\mathrm{H} 2$ antagonist ranitidine on the absorption of BAY 59-7939 (rivaroxaban., an oral, direct Factor Xa inhibitor, in healthy subjects. $J$ Clin Pharmacol, 46:549-58.

Lassen MR, Ageno W, Borris LC, et al. 2008. Rivaroxaban versus enoxaparin for thromboprophylaxis after total knee arthroplasty. $N$ Engl J Med, 358:2776-86.
Lassen MR, Davidson BL, Gallus A, et al. 2006. On behalf of the apixaban investigators. A phase II randomized, double-blind, eight-arm, parallel-group, dose-response study of apixaban, a new oral Factor Xa inhibitor for the prevention of deep vein thrombosis in knee replacement surgery. Blood, 108:Abstract 574.

Lassen MR, Davidson BL, Gallus A, et al. 2007. The efficacy and safety of apixaban, an oral, direct factor Xa inhibitor, as thromboprophylaxis in patients following total knee replacement. J Thromb Haemost, 5:2368-75.

Lassen MR, Davidson BL, Gallus AS, et al. 2003. A phase II randomized, double-blind, five-arm, parallel-group, dose-response study of a new oral directly-acting Factor Xa inhibitor, razaxaban, for the prevention or deep vein thrombosis in knee replacement surgery. Blood, 102:Abstract 41 .

Linder R, Blomback M, Egberg N, et al. 1999. Thrombin inhibitors suppress the thrombin-thrombomodulin-mediated generation of activated protein C. Thromb Res, 95:117-25.

Mann KG, Brummel K, Butenas S. 2003. What is all that thrombin for? $J$ Thromb Haemost, 1:1504-14.

McBride BF. 2005. A preliminary assessment of the critical differences between novel oral anticoagulants currently in development. $J$ Clin Pharmacol, 45:1004-17.

McBride D, Bruggenjurgen B, Roll S, et al. 2007. Anticoagulation treatment for the reduction of stroke in atrial fibrillation: a cohort study to examine the gap between guidelines and routine medical practice. $J$ Thromb Thrombolysis, 24:65-72.

Menzin J, Boulanger L, Hauch O, et al. 2005. Quality of anticoagulation control and costs of monitoring warfarin therapy among patients with atrial fibrillation in clinic settings: a multi-site managed-care study. Ann Pharmacother, 39:446-51.

Morishima Y, Furugohri T, Isobe K, et al. 2004. In vitro characteristics, anticoagulant effects and in vivo antithrombotic efficacy of a novel, potent and orally active direct factor Xa inhibitor, DU-176b. Blood, 104:Abstract 1862.

Oake N, Fergusson DA, Forster AJ, et al. (2007. Frequency of adverse events in patients with poor anticoagulation: a meta-analysis. CMAJ, 176:1589-94.

Olsson SB. 2003. Stroke prevention with the oral direct thrombin inhibitor ximelagatran compared with warfarin in patients with non-valvular atrial fibrillation (SPORTIF III.: randomised controlled trial. Lancet, 362:1691-8

Roehrig S, Straub A, Pohlmann J, et al. 2005. Discovery of the novel antithrombotic agent 5-chloro-N-(\{(5S)-2-oxo-3- [4-(3-oxomorpholin4-yl)phenyl]-1,3-oxazolidin-5-yl\}methyl)thiophene- 2-carboxamide (BAY 59-7939): an oral, direct Factor Xa inhibitor. J Med Chem, 48:5900-8.

Schulman S, Wahlander K, Lundstrom T, et al. 2003. Secondary prevention of venous thromboembolism with the oral direct thrombin inhibitor ximelagatran. $N$ Engl J Med, 349:1713-21.

Stangier J, Eriksson BI, Dahl OE, et al. 2005. Pharmacokinetic profile of the oral direct thrombin inhibitor dabigatran etexilate in healthy volunteers and patients undergoing total hip replacement. $J$ Clin Pharmacol, 45:555-63.

Stangier J, Rathgen K, Stahle H, et al. 2007. The pharmacokinetics, pharmacodynamics and tolerability of dabigatran etexilate, a new oral direct thrombin inhibitor, in healthy male subjects. Br J Clin Pharmacol, 64:292-303.

Tarzami ST, Wang G, Li W, et al. 2006. Thrombin and PAR-1 stimulate differentiation of bone marrow-derived endothelial progenitor cells $J$ Thromb Haemost, 4:656-63.

The RE-MOBILIZE Writing Committee 2008. The oral thrombin inhibitor dabigatran etexilate vs the north american enoxaparin regimen for the prevention of venous thromboembolism after knee arthroplasty surgery. J Arthroplasty, Doi:10.1016/j.arth.2008.01.132.

Turpie AG. 2007. Oral, direct Factor Xa inhibitors in development for the prevention and treatment of thromboembolic diseases. Arterioscler Thromb Vasc Biol 27:1238-47. 
Turpie AGG, Bauer KA, Davidson BL, et al. 2008. Comparison of rivaroxaban - an oral, direct Factor Xa inhibitor - and subcutaneous enoxaparin for thromboprophylaxis after total knee replacement (RECORD4: a phase III study). European Federation of National Associations of Orthopaedics and Traumatology 2008 Annual Meeting; May 29-June 1, 2008; Nice, France Abstract F85 .

Turpie AGG, Fisher WD, Bauer K, et al. 2005. An oral, direct Factor Xa inhibitor - BAY 59-7939 - for prophylaxis against venous thromboembolism after total knee replacement: a phase II dose-ranging study. J Thromb Haemost, 3:2479-86.

Vesey DA, Cheung CW, Kruger WA, et al. 2005. Thrombin stimulates proinflammatory and proliferative responses in primary cultures of human proximal tubule cells. Kidney Int, 67:1315-29.
Warkentin TE, Levine MN, Hirsh J, et al. 1995. Heparin-induced thrombocytopenia in patients treated with low-molecular-weight heparin or unfractionated heparin. $N$ Engl J Med, 332:1330-5.

Weinz C, Schwartz T, Pleiss U et al. 2004. Metabolism and distribution of $\left[{ }^{14} \mathrm{C}\right]$ BAY 59-7939 - an oral, direct Factor Xa inhibitor - in rat, dog and human. Drug Metab Rev, 36:Abstract 196.

Weitz JI. 2006. Emerging anticoagulants for the treatment of venous thromboembolism. Thromb Haemost, 96:274-84.

Zafar MU, Vorchheimer DA, Gaztanaga J, et al. 2007. Antithrombotic effects of factor Xa inhibition with DU-176b: Phase-I study of an oral, direct factor Xa inhibitor using an ex-vivo flow chamber. Thromb Haemost, 98:883-8. 